\title{
Complementary cooperation between two syntrophic bacteria in pesticide degradation
}

\author{
Chie Katsuyama $^{a}$, Shinji Nakaoka ${ }^{b, c}$, Yasuhiro Takeuchi ${ }^{a}$, \\ Kanako Tago $^{d}$, Masahito Hayatsu ${ }^{d}$, and Kenji Kato ${ }^{a, *}$
}

${ }^{a}$ Department of Environment and Energy System, Graduate School of Science and Technology, Shizuoka University, 836 Ohya, Suruga-ku, Shizuoka 422-8529, Japan,

${ }^{b}$ JST ERATO Aihara Complexity Modelling Project, The University of Tokyo, 46-1 Komaba, Meguro-ku, Tokyo 153-8505, JAPAN,

${ }^{c}$ Graduate School of Mathematical Sciences, The University of Tokyo 3-8-1, Komaba, Meguro-ku, Tokyo, 153-8914, Japan,

$d$ Department of Environmental Science for Human Life, Faculty of Agriculture, Shizuoka University, 836 Ohya, Suruga-ku, Shizuoka 422-8529, Japan,

${ }^{e}$ Department of Geosciences, Faculty of Science, Shizuoka University, 836 Ohya, Suruga-ku, Shizuoka 422-8529, Japan

* Corresponding author. Department of Geosciences, Faculty of Science, Shizuoka University, 836 Ohya, Suruga-ku, Shizuoka 422-8529, Japan. Tel.: + 8154238 4950; fax: + 8154238 4950. E-mail address: skkato@ipc.shizuoka.ac.jp

\begin{abstract}
Interactions between microbial species, including competition and mutualism, influence the abundance and distribution of the related species. For example, metabolic cooperation among multiple bacteria plays a major role in the maintenance of consortia. This study aims to clarify how two bacterial species coexist in a syntrophic association involving the degradation of the pesticide fenitrothion. To elucidate essential mechanisms for maintaining a syntrophic association, we employed a mathematical model based on an experimental study, because experiment cannot elucidate various conditions for two bacterial coexistence. We isolated fenitrothion-degrading Sphingomonas sp. TFEE and its metabolite of 3-methyl-4-nitrophenol (3M4N)-degrading Burkholderia sp. MN1 from a fenitrothion-treated soil microcosm. Neither bacterium can completely degrade fenitrothion alone, but they can utilize the second intermediate, methylhydroquinone (MHQ). Burkholderia sp. MN1 excretes a portion of MHQ during the degradation of 3M4N, from which Sphingomonas sp. TFEE carries out degradation to obtain carbon and energy. Based on experimental findings, we developed mathematical models that represent the syntrophic association involving the two bacteria. We found that
\end{abstract}


the two bacteria are characterized by the mutualistic degradation of fenitrothion. Dynamics of two bacteria are determined by the degree of cooperation between two bacteria (i.e., supply of 3M4N by Sphingomonas sp. TFEE and excretion of MHQ by Burkholderia sp. MN1) and the initial population sizes. The syntrophic association mediates the coexistence of the two bacteria under the possibility of resource competition for MHQ, and robustly facilitates the maintenance of ecosystem function in terms of degrading xenobiotics. Thus, the mathematical analysis and numerical computations based on the experiment indicate the key mechanisms for coexistence of Sphingomonas sp. TFEE and Burkholderia sp. MN1 in syntrophic association involving fenitrothion degradation.

Keywords: Microbial consortia; Mathematical modeling; Experimental findings; Syntrophic association

\section{Introduction}

Bacteria distribute everywhere on the Earth, and their biomass is estimated to be equal to or even exceed that of plants (Whitman et al., 1998). In a given system, bacteria exist at a high density, and the community comprises numerous clones (species) and various functional groups (Torsvik et al., 2002a,b; Gans et al., 2005). Metabolically related bacterial populations occur within a given space. Indeed, some bacterial populations correlate strongly with each other in the expression of function (e.g., Overmann et al., 2002); such an association is regarded as a microbial consortium. The microbial consortia, thus being a fundamental component of an ecosystem, may demarcate biodiversity and ecosystem function.

One of the mechanisms for maintaining microbial consortia is syntrophic association: a process whereby two or more microorganisms cooperate to degrade a substrate or substrates that neither can degrade alone (Madigan et al., 2000; Keller et al., 2004). Under the syntrophic association, the fitness of each partner positively depends on the fitness of the other. These microorganisms depend on one another for metabolic products or maintenance of chemically permissive conditions. Syntrophic association have been demonstrated in several ecosystems, for example, interspecies hydrogen transfer in methanogenic degradation (Schink, 1997), archaean-bacterium partnerships that link methane oxidation and sulfate-reduction (Valentine, 2002) or denitrification (Raghoebarsing et al., 2006), and xenobiotic degradation (Dejonghe et al., 2003; Smith et al., 2005).

The advantage of studying a xenobiotics-degrading consortium as a model for investigating the role of syntrophic association in microbial communities is that the number of species associated with the degradation of xenobiotics is relatively small compared with the number involved in degrading a natural organic compound in a given environment. The exposure of soil microbes to xenobiotics commonly leads to a functional shift in the ecosystem associated with the replacement of the dominant 
bacterial species by those with different traits that confer the ability to degrade the introduced xenobiotics. Thus the introduction of xenobiotics may develop the novel interactions between bacterial species. We are therefore able to establish a simple environment in which to study syntrophic association. It is often the case that more than two species are synergistically involved in the degradation of xenobiotics by syntrophic association or cross-feeding in a xenobiotics-degrading consortium (Dejonghe et al., 2003; Smith et al., 2005). Although a diverse range of studies have investigated xenobiotics-degrading consortia where syntrophic associations play an important role (Alonso et al., 1997; Dejonghe et al., 2003; Smith et al., 2005), our current understanding of microbial consortia is limited to descriptions of the members and their inherent functions. The manner of the interaction required to maintain a syntrophic association of two or more species in a given metabolic sequence has yet to be elucidated.

In case of intraspecies interaction, various theories have been presented to address the question of how cross-feeding, which is very similar to syntrophic association though it is not necessarily mutualism, evolves in microbial communities. Pfeiffer et al. (2004) showed how stable polymorphisms are maintained by cross-feeding as a consequence of optimization principles. Doebeli (2002) proposed a theory that explains how cross-feeding polymorphisms can emerge from a monomorphism, based on a framework of adaptive dynamics. Many experimental studies have investigated the adaptive diversification of $E$. coli populations (Rosenzweig et al., 1994; Friesen et al., 2004). Rainey et al. (2003) showed empirically the evolution of cooperation in bacterial communities via a diversification of morphology. Such cooperative activity might determines the diversity of species in a community. Although these previous studies clearly demonstrate the feasibility of cross-feeding in microbial communities as a consequence of evolution, the emergence and maintenance mechanism of syntrophic association in a microbial consortium has yet to be fully investigated. In case of interspecies interaction, it was shown by mathematical modeling that excretion of intermediate substrate facilitates commensal interactions which enable competitive species to coexist (Bratbak et al., 1995; Zissi et al., 2001; Miki et al., 2005). The mechanism of mutualistic interspecies interaction to sustain coexistence is scarcely elucidated from the stand point of population dynamics of bacteria constituting a syntrophic association.

In this paper, we report mathematical solution on the sustainable mechanism of the smallest subset of an obligately syntrophic bacterial consortium, consisting of two bacteria: Sphingomonas sp. TFEE and Burkholderia sp. MN1. These bacteria were isolated from soil microcosms treated with the pesticide fenitrothion [O,O-dimethyl $O$-(4-nitro- $m$-tolyl) phosphorothioate]. Although fenitrothion was completely degraded by these bacteria, neither is capable of degrading fenitrothion alone. If syntrophic association of two bacteria occur in the fenitrothion-treated soil, they were able to grow on the intermediate products. It is not practical to carry out long-term experiments of fenitrothion degradation repeatedly and to examine various combinations of conditions. Thus, to compensate those difficulties 
of experimental microbiology, mathematical analysis is employed to understand the sustainable mechanisms using a representative example of syntrophic association taken from the experiment.

We developed mathematical models that represent the manner of interaction in the syntrophic association of the two bacteria. The intermediate methylhydroquinone (MHQ) is a common resource for Sphingomonas sp. TFEE and Burkholderia sp. MN1, suggesting the occurrence of exploitative competition for MHQ between these bacteria. The competitive exclusion principle states that a single limiting resource can support only a single competitor (Armstrong et al., 1980; Smith et al., 1994; Grover et al., 1997). It is notable that Burkholderia sp. MN1 excretes MHQ to the surrounding environment. Our study addresses the question of how a syntrophic association mediates the coexistence of two phylogenetically distant bacteria in a given soil under the possibility of exploitative competition for MHQ.

\section{Experimental Results}

\subsection{Isolation of the fenitrothion-degrading bacterium TFEE and the 3M4N-degrading bacterium MN1 from soil}

Repeated applications of fenitrothion to soil microcosms led to the development of fenitrothion-degrading microbial populations. The number of fenitrothion-degrading bacteria increased from day 42 , amounting to $1.2 \times 10^{7}$ colony-forming units (CFU) $\mathrm{g}^{-1}$ of dry soil at day 396 (Fig. 1). We successfully isolated predominant two degraders from the treated soil directly. The first degrader, a fenitrothion-degrading bacterium, was designated TFEE. The second degrader, a 3M4N-degrading bacterium, was designated MN1. The $16 \mathrm{~S}$ rRNA gene sequence showed TFEE and MN1 was preliminarily identified to be the genera Sphingomonas and Burkholderia, respectively.

\subsection{Degradation of fenitrothion and its metabolites}

Both Sphingomonas sp. TFEE and Burkholderia sp. MN1 lacked the complete pathway of fenitrothion degradation. Sphingomonas sp. TFEE degraded fenitrothion to produce an almost stoichiometric amount of 3M4N. However, it did not degrade 3M4N. Thus 3M4N accumulated (the first step in Fig. 2) and Sphingomonas sp. TFEE was unable to grow on fenitrothion without the collaboration of Burkholderia sp. MN1. Burkholderia sp. MN1 degraded 3M4N and grew on it as a sole carbon source, though it was unable to degrade fenitrothion to $3 \mathrm{M} 4 \mathrm{~N}$. In mono-culture with 3M4N, Burkholderia sp. MN1 utilized MHQ and excreted a portion of MHQ by the degradation of 3M4N. MHQ transiently accumulated MHQ in the medium (the second step in Fig. 2). Excreted MHQ can be utilized by Sphingomonas sp. TFEE (the third step in Fig. 2). Grown Sphingomonas sp. TFEE based on MHQ, 
thus degrades fenitrothion if MHQ is continuously supplied.

\subsection{Co-culture of two bacteria with MHQ or fenitrothion}

Both Sphingomonas sp. TFEE and Burkholderia sp. MN1 increased based on MHQ incubated either mono-culture or co-culture (Fig. 3). After $25 \mathrm{~h}$ of co-culture based on MHQ with yeast extract, both bacteria increased about five times higher than those co-cultured with yeast (Table 1). In case of mono-culture, the growth of Sphingomonas sp. TFEE in medium with MHQ was three times higher than that of Burkholderia sp. MN1 (Table 1), indicating that Sphingomonas sp. TFEE shows a higher yield based on MHQ than that of Burkholderia sp. MN1. Therefore, theoretically, competition for MHQ may occur between two bacteria. However, Burkholderia sp. MN1 excretes surplus MHQ which is swiftly utilized by Sphingomonas sp. TFEE. The results of these experiments suggest the degree of excreted MHQ may be the key mechanism for the continuous coexistence of two bacteria.

Sphingomonas sp. TFEE and Burkholderia sp. MN1 were initially inoculated at $1 \times 10^{6} \mathrm{CFU} \mathrm{ml}^{-1}$ and $1 \times 10^{8} \mathrm{CFU} \mathrm{ml}^{-1}$, respectively, into the medium added with fenitrothion as a sole carbon source. Neither bacteria grew in the medium after $48 \mathrm{~h}$ of incubation (data not shown). Increased inoculation density of Sphingomonas sp. TFEE to $1 \times 10^{7} \mathrm{CFU} \mathrm{ml}^{-1}$ enabled Burkholderia sp. MN1 to grow in the medium, suggesting initial number of Sphingomonas sp. TFEE constrained the growth of Burkholderia sp. MN1.

Taken together, we found the two bacteria Sphingomonas sp. TFEE and Burkholderia sp. MN1 have complementary degradation machinery (Fig. 2). The results of these experiments suggest the key mechanisms for coexistence of two bacteria: the amount of excreted MHQ constraining the number of Sphingomonas sp. TFEE, and the initial population size and the fenitrothion-degrading rate of Sphingomonas sp. TFEE constraining the number of Burkholderia sp. MN1. To understand the variety of these conditions and to elucidate how two bacterial species coexist in soil and how stable the coexistence is, we developed mathematical models based on the experimental findings.

\section{Mathematical Analysis}

\subsection{Model formulation}

Our model represents the population dynamics of two bacteria, Sphingomonas sp. TFEE and Burkholderia sp. MN1, in fenitrothion-treated soil (Fig. 2 and Table 2). The variables $S_{0}, S_{1}$, and $S_{2}$ denote the concentrations of fenitrothion, 3M4N, and MHQ in the soil, respectively. The population densities of Sphingomonas sp. TFEE and Burkholderia sp. MN1 are denoted by $x_{1}$ and $x_{2}$, respectively. The repeated application of fenitrothion is represented by $\lambda$. Although bacterial degradation is considered to be a major factor determining the fate of fenitrothion, $3 \mathrm{M} 4 \mathrm{~N}$, and 
MHQ, these compounds naturally decompose over time, as represented by $-d_{0} S_{0}$, $-d_{1} S_{1}$, and $-d_{2} S_{2}$, respectively. We do not consider any possibilities that metabolite $3 \mathrm{M} 4 \mathrm{~N}$ and MHQ are produced via natural decomposition of fenitrothion and 3M4N.

We assume that the uptake rate of $3 \mathrm{M} 4 \mathrm{~N}$ and MHQ follows the conventional formulation known as Michaelis-Menten kinetics, with a half-saturation constant $a_{j}$ and maximum uptake rate $m_{j}$ of the form: $f_{j}\left(S_{i}\right):=\frac{m_{j} S_{i}}{a_{j}+S_{i}},(i, j=1,2)$. Population growth of the bacteria is simply proportional to the uptake of substrates with constant conversion rates (i.e., yields) of $\eta_{1}$ for Sphingomonas sp. TFEE and $\eta_{2}$ for Burkholderia sp. MN1, respectively. Conventionally we can assume that interaction between enzyme and substrate follows the mass action law (Keener et al., 1998). Let $E(t)$ denote the concentration of the fenitrothion-degrading enzyme synthesized by Sphingomonas sp. TFEE. We assume that $E(t)$ is proportional to the population density of Sphingomonas sp. TFEE, that is, $E(t)=k x_{1}(t)$. Hence the concentration of enzyme-substrate complex is given by $\beta^{\prime} S_{0} E:=\beta S_{0} x_{1}$. Here constant $\beta=k \beta^{\prime}$ represents the rate of decomposition of enzyme-substrate complex into $3 \mathrm{M} 4 \mathrm{~N}$ and enzyme. Following the experimental observations, we assume that a portion of MHQ is excreted from Burkholderia sp. MN1 after the metabolism of 3M4N. Let $\gamma$ denote the fraction of MHQ excreted by Burkholderia sp. MN1. By definition, $\gamma$ must be assigned a value between 0 and 1 . Hence the total excretion of MHQ is given by $\gamma f_{2}\left(S_{1}\right) x_{2} / \eta_{2}$. The remaining $(1-\gamma) f_{2}\left(S_{1}\right) x_{2} / \eta_{2}$ is exploited for the population growth of Burkholderia sp. MN1. Loss of activity for individual bacterium can occur via starvation, mutation, being prey to predation, or other factors. We simply assume that the loss rates of Sphingomonas sp. TFEE and Burkholderia sp. MN1 are given by $-\mu_{1} x_{1}$ and $-\mu_{2} x_{2}$, respectively. The model, including all of the above assumptions, is described by the following set of differential equations:

$$
\begin{aligned}
\frac{d S_{0}}{d t} & =\lambda-d_{0} S_{0}-\beta S_{0} x_{1} \\
& =(\text { input })-(\text { decomposition })-(\text { degradation }), \\
\frac{d S_{1}}{d t} & =-d_{1} S_{1}+\beta S_{0} x_{1}-f_{2}\left(S_{1}\right) \frac{x_{2}}{\eta_{2}} \\
& =-(\text { decomposition })+(\text { production })-(\text { uptake }), \\
\frac{d S_{2}}{d t} & =-d_{2} S_{2}+\gamma f_{2}\left(S_{1}\right) \frac{x_{2}}{\eta_{2}}-f_{1}\left(S_{2}\right) \frac{x_{1}}{\eta_{1}} \\
& =-(\text { decomposition })+(\text { excretion })-(\text { uptake }), \\
\frac{d x_{1}}{d t} & =x_{1}\left(f_{1}\left(S_{2}\right)-\mu_{1}\right) \\
& =(\text { growth })-(\text { loss }), \\
\frac{d x_{2}}{d t} & =x_{2}\left((1-\gamma) f_{2}\left(S_{1}\right)-\mu_{2}\right) \\
& =(\text { growth })-(\text { loss }) .
\end{aligned}
$$

As Burkholderia sp. MN1 utilizes MHQ before it is excreted, our experimental re- 
sult suggests that the excreted MHQ is less expected to be utilized by Burkholderia sp. MN1 once again. Therefore we neglect the uptake of MHQ by Burkholderia sp. MN1 in (Eq). We can show that qualitatively the same consequence is derived even though Burkholderia sp. MN1 is capable of utilizing the MHQ excreted by itself (see Appendix B.4). Exact parameter values for the equation (Eq) were practically difficult to determine from the experiment with soil microcosm, since the experiment had continued over one year. The default values of parameters for numerical computations and calculations are given by $\lambda=0.1, d_{0}=0.05, m_{1}=1.5, m_{2}=2.5$, $a_{1}=1.1, a_{2}=2.0, \mu_{1}=0.01, \mu_{2}=0.02, \eta_{1}=0.5$ and $\eta_{2}=0.2$ (see Table 1 for the units of parameters). We neglect the difference in natural decomposition rate among fenitrothion and its metabolites. This yields $d_{0}=d_{1}=d_{2}=0.05$. The parameters $\beta$ and $\gamma$ are controlled to examine how the sustainable degradation of fenitrothion is mediated.

\subsection{Analysis and results}

There are three possible equilibria in our mathematical model. Null-degradation equilibrium $E_{N}=\left(\frac{\lambda}{d_{0}}, 0,0,0,0\right)$ always exists. All substrates and bacteria except fenitrothion are diminished at this equilibrium. $E_{N}$ is stable when the degradation of fenitrothion does not occur. Mathematically, it is easy to show that $E_{N}$ is always locally stable (see Appendix B.2). This implies that the population densities of two bacteria at the initiation of degradation can determine the outcome whether fenitrothion is successfully degraded or not. The sustainable degradation of fenitrothion requires the existence of at least one stable positive equilibrium. It is easy to see that $x_{1}=0$ if and only if $x_{2}=0$ at an equilibrium. Hence there is no equilibrium at which only one bacterium can exist. In other words, there is no possibility of competitive exclusion between these two bacteria in our system.

Hereafter we will show that there are two positive equilibria in general. One is Syntrophic consortium equilibrium $E_{S}:=\left(S_{0}^{+}, S_{1}^{*}, S_{2}^{*}, x_{1}^{+}, x_{2}^{+}\right)$which represents the successful degradation of fenitrothion and the stable coexistence of two bacteria (see Appendix B.2). Another one is unstable equilibrium $E_{U}:=\left(S_{0}^{-}, S_{1}^{*}, S_{2}^{*}, x_{1}^{-}, x_{2}^{-}\right)$ which induces the threshold associated with the initial population size by which it is determined whether two bacteria coexist or not. The existence of these positive equilibria is determined by the following equation with respect to $x_{1}$ :

$$
\frac{\lambda \gamma \beta x_{1}}{d_{0}+\beta x_{1}}=\frac{\mu_{1}}{\eta_{1}} x_{1}+\gamma d_{1} S_{1}^{*}+d_{2} S_{2}^{*}
$$

where $S_{1}^{*}$ and $S_{2}^{*}$ are respectively given by $a_{2} \mu_{2} /\left\{m_{2}(1-\gamma)-\mu_{2}\right\}$ and $a_{1} \mu_{1} /\left(m_{1}-\mu_{1}\right)$ whenever $m_{2}>\mu_{2} /(1-\gamma)$ and $m_{1}>\mu_{1}$. It follows from (1) that the syntrophic consortium equilibrium is likely to exist when $\beta$ is sufficiently large (see Appendix Fig. 7). Recall that $\beta$ denotes the rate of decomposition of enzyme-substrate complex into 3M4N and enzyme. Therefore active degradation of fenitrothion mediated by Sphingomonas sp. TFEE is important to maintain the syntrophic association. 
It also follows from (1) that there is no syntrophic consortium equilibrium when $\gamma=0$. Hence there must be a minimum value of $\gamma$, denoted by $\gamma_{\min }$, such that the condition $\gamma>\gamma_{\min }$ is required for the establishment of the syntrophic association. Recall that $\gamma$ denotes the fraction of MHQ excreted by Burkholderia sp. MN1. Therefore Burkholderia sp. MN1 would obtain the maximum carbon gain by utilizing 3M4N when no MHQ is excreted, i.e., $\gamma=0$. The condition for the existence of the syntrophic consortium equilibrium indicates that the maintenance of the syntrophic association requires the minimum effort by Burkholderia sp. MN1. These verbal notations for the existence of the syntrophic consortium equilibrium are summarized as follows (mathematical precise conditions are derived in Appendix B.1):

Condition for the maintenance of individuals: $m_{1}>\mu_{1}$ and $m_{2}>\mu_{2} /(1-\gamma)$ Condition for the maintenance of consortium: large $\beta$ (contribution by TFEE) and $\gamma_{\min }<\gamma$ (contribution by MN1).

Note that the default parameters are one example of a successful syntrophic association. The syntrophic association can be established for various another sets of parameters. These steady state analyses indicate that the dynamics exhibited by (Eq) may depend not only on the two parameters $\beta$ and $\gamma$ representing the cooperation between them, but also on the initial population size of these bacteria. Hereafter we shall perform numerical calculations to examine how the syntrophic association is organized depending on the initial population sizes $x_{j}(0)(j=1,2)$ and the two parameters $\beta$ and $\gamma$. At the beginning of the experiment in soil microcosm, the initial population density of Sphingomonas sp. TFEE was below the detection limit, $10^{3}$ cells $\mathrm{g}^{-1}$ (Fig. 1). In numerical calculations, we assume that the default values of initial population sizes for Sphingomonas sp. TFEE and Burkholderia sp. MN1 are given at the threshold of the detectable level; $x_{1}(0)=x_{2}(0)=0.0001 \equiv 10^{3}$ cells $\mathrm{g}^{-1}$, respectively. All numerical calculations are implemented with fourth-order Runge-Kutta method. Fig. 4A depicts the dynamics of two bacteria with $\beta=50$ and $\gamma=0.3$. Although it takes a long time to reach a significant level of population size, two bacteria successfully grow in number. Mathematically, the densities of two bacteria converge to syntrophic consortium equilibrium $E_{S}$. In order to investigate the dependence of the dynamics on the fraction of MHQ excretion $\gamma$, we perform numerical calculations with $\gamma=0.7$ under the unchanged other parameters. In this case, Burkholderia sp. MN1 excretes a large fraction of MHQ. In contrast to the case in Fig. 4A, both of two bacteria do not exhibit any significant growth and eventually decline to below the detectable level (see Fig. 4B). An over-excretion of MHQ by Burkholderia sp. MN1 may be beneficial to Sphingomonas sp. TFEE but detrimental to Burkholderia sp. MN1 for its existence. We shall investigate the opposite situation to the previous case in which Burkholderia sp. MN1 do not provide sufficient MHQ to Sphingomonas sp. TFEE. The result is depicted in Fig. 
$4 \mathrm{C}$ which is obtained by performing numerical calculations with $\gamma=0.1$. Interestingly, only Burkholderia sp. MN1 substantially grow for a long time. However, eventually it declines toward the undetectable level. This long-term transient survival of Burkholderia sp. MN1 can be explained as follows. Burkholderia sp. MN1 obtains sufficient carbon from 3M4N, while it excreted a very low amount of MHQ. Thus, Sphingomonas sp. TFEE, producing 3M4N, can not obtain sufficient MHQ as a carbon source because of its little excretion by Burkholderia sp. MN1. Finally Burkholderia sp. MN1 suffers from the lack of 3M4N due to the absence of Sphingomonas sp. TFEE. This numerical calculation result is quite well in agreement with the experimental result; only the growth of Burkholderia sp. MN1 was observed in the fenitrothion-treated medium (see Experimental results 2.3). The insight to this experimental observation from mathematical analyses is that Burkholderia sp. MN1 might not excrete sufficient MHQ in the fenitrothion-treated medium. Note that the establishment of the syntrophic association was in fact observed in a soil microcosm. Hence it is expected that Burkholderia sp. MN1 may excrete sufficient amount of MHQ to sustain the abundance of Sphingomonas sp. TFEE in the consortium.

Next, we examine whether the augmentation of bacteria can facilitate the establishment of the syntrophic association. We shall vary the initial population size of Sphingomonas sp. TFEE, while keeping the same initial population size for Burkholderia sp. MN1 under the condition that the values of parameters remain unchanged from the case depicted in Fig. $4 \mathrm{C} ; \beta=50$ and $\gamma=0.1$. Fig. 4D

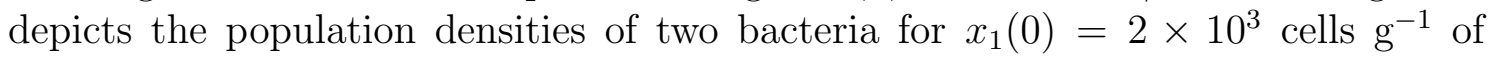
Sphingomonas sp. TFEE. With the augmentation of Sphingomonas sp. TFEE, it becomes possible to achieve the successful degradation mediated by the syntrophic association (see Fig. 4D). This numerical calculation result suggests that bistability between two steady states, successful or unsuccessful degradation of fenitrothion, may generically occur. To confirm this view, we draw a diagram with respect to the initial population sizes of two bacteria. The diagram depicted in Fig. 5 shows two qualitatively different outcomes. A saddle type unstable equilibrium $E_{U}$ may divide the region into two parts, namely, associative region and dissociative region (Fig. 5). On the associative region (shown by green in Fig. 5), all solutions tend to syntrophic equilibrium $E_{S}$, while on the dissociative region (shown by white in Fig. 5), all solutions tend to null-degradation equilibrium $E_{N}$. Bistability between $E_{N}$ and $E_{S}$ is a common feature.

The effect of fenitrothion degradation by Sphingomonas sp. TFEE $(\beta)$ and MHQ excretion by Burkholderia sp. MN1 $(\gamma)$ at the syntrophic consortium equilibrium is investigated by varying $\beta$ and $\gamma$. Note that the equilibrium concentration of MHQ is independent of $\beta$ and $\gamma$. Therefore we prepare four cases which depict the densities of two bacteria and the concentrations of fenitrothion and 3M4N (Fig. 6). For example, syntrophic association is established for $\gamma$ assigned in the admissible excretion range $[0.151,0.975]$ if $\beta=0.1$. Note that the syntrophic consortium equilibrium can exist even for $\beta=0.05$ which seems very small compared to $\beta=50$ employed in our numerical calculations. The existence of the syntrophic consortium equilibrium 
does not necessarily imply the successful degradation of fenitrothion. The successful degradation additionally requires sufficiently large population size or another factor as the accumulation of fenitrothion. In fact, additional numerical calculations with $\gamma=0.3$ and $x_{1}(0)=x_{2}(0)=1.0 \times 10^{3}$ cells $\mathrm{g}^{-1}$ indicate that the degradation of fenitrothion is successful for $\beta \geq 37$ if $S_{0}(0)=1, \beta \geq 26$ if $S_{0}(0)=5$ and $\beta \geq 10$ if $S_{0}(0)=10$ (figures not shown). Hence the accumulation of fenitrothion may trigger the establishment of the syntrophic association. Although model (Eq) does not consider the accumulation of the enzyme to decompose fenitrothion synthesized by Sphingomonas sp. TFEE, accumulation of this enzyme can promote the degradation of fenitrothion. The degradation of fenitrothion is successful for $\beta \geq 4$ if the initial population size of two bacteria is large such that $x_{1}(0)=x_{2}(0)=1.0 \times 10^{4}$ cells g $^{-1}$. Hence large initial population size allows the establishment of syntrophic association more easier. Notably, the admissible excretion range $\left[\gamma_{\min }, \gamma_{\max }\right]$ increases with increasing $\beta$ (Fig. 6). Thus, the cooperative activity of Sphingomonas sp. TFEE promotes the cooperative activity of Burkholderia sp. MN1. The maximum density of Sphingomonas sp. TFEE is attained near $\gamma_{\max }$, while the maximum density of Burkholderia sp. MN1 is attained near $\gamma_{\min }$, implying the existence of constraint on the growth of two bacteria. For large $\beta$, both fenitrothion and 3M4N are effectively reduced. Fenitrothion can be more effectively reduced for values of $\gamma$ close to $\gamma_{\max }$. The concentration of fenitrothion is minimized when the density of Sphingomonas sp. TFEE is maximized. The concentration of $3 \mathrm{M} 4 \mathrm{~N}$ shows a monotonic increase with $\gamma$.

By mathematical analyses and numerical computations based on the experimental study, we identified three important mechanisms that underlie the long term coexistence of the two bacteria. The first key mechanism is a trade-off in resource utilization. Despite the fact that two bacteria grew on the experiment treated with MHQ during 25 hours, most theoretical studies predict that eventually one of species will outcompete the other in exploitative competition for the limiting resource MHQ (Armstrong et al., 1980; Smith et al., 1994; Grover et al., 1997). In our mathematical investigation for the growth of two bacteria on MHQ-treated media, we predict that only Sphingomonas sp. TFEE may eventually survive if long-term experiment more than 25 hours is performed (see Appendix B.3 for details). Hence the establishment of syntrophic association gives rise to the mechanism of avoiding or relaxing resource competition.

The second key mechanism is cooperative activity by Burkholderia sp. MN1. The value of $\gamma$ must be assigned between the threshold values of $\gamma_{\min }$ and $\gamma_{\max }$. A moderate fraction of MHQ excretion by Burkholderia sp. MN1 is necessary for sustainable coexistence of two bacteria. In the extreme case of $\gamma=1$, we can show that Burkholderia sp. MN1 cannot be sustainably maintained because of no carbon gain. Hence, the maximum excretion ratio $\gamma_{\max }$ is required for the growth of Burkholderia sp. MN1. Conversely, if $\gamma=0$, no excretion of MHQ results in the breakdown of successive cooperation; in other words, the minimum excretion ratio $\gamma_{\min }$ is required to maintain the syntrophic association. The existence of the lowest 
fraction of MHQ excretion can be viewed as a cooperative activity by Burkholderia sp. MN1, because Burkholderia sp. MN1 would obtain the maximum benefit when $\gamma=0$.

The third key mechanism is cooperative activity by Sphingomonas sp. TFEE. A large value of $\beta$ implies the active decomposition of enzyme-substrate complex. In other words, the active cooperative degradation of fenitrothion is required for their sustainable coexistence. These observations suggest that the syntrophic association is maintained by complementary degradation.

\section{Discussion}

Based on experimental findings, we employed mathematical analysis to understand the substantial mechanisms of syntrophic association in degradation of pesticide, as it is not practical to elucidate various combinations of conditions in bacterial interactions. We found a mutualistic consortium consisting of Sphingomonas sp. TFEE and Burkholderia sp. MN1 that degrades fenitrothion cooperatively in fenitrothiontreated soil (Fig. 2) These two bacteria are characterized by mutual complementation: both have complementary functions that enable the complete degradation of fenitrothion. Mutualistic degradation provides a means to avoid the overlap of niche, by which two bacteria can stably coexist. There exists a trade-off between resource utilization of 3M4N and MHQ, where Sphingomonas sp. TFEE utilizes MHQ while Burkholderia sp. MN1 utilizes both 3M4N and MHQ. The finding from experiment suggests that the function of excretion of surplus MHQ by Burkholderia sp. MN1 sustained the abundance of Sphingomonas sp. TFEE to keep coexistence.

The essential mechanisms for the coexistence of two bacteria in soil can be understood by the simple mathematical model. The dynamics of two bacterial populations depends on two parameters $\beta$ (Fig. 7) and $\gamma$ (Fig. 4A-C) that represent the cooperation between them and on their initial population sizes (Fig. 4C,D and Fig. 5). The variety of $\beta$ and $\gamma$ at the syntrophic consortium equilibrium (Fig. 6) indicates how two bacteria coexist in a syntrophic association. It is supposed that $\beta$ of Sphingomonas sp. TFEE in soil should be sufficiently large to coexist with Burkholderia sp. MN1, though the activity of fenitrothion degradation of Sphingomonas sp. TFEE varies with environmental conditions (data not shown). In fact, Sphingomonas sp. TFEE is superior in fenitrothion degradation to Burkholderia sp. NF100 (Hayatsu et al., 2000)(data not shown) that is the single complete degrader. The degradative ability of one bacterium facilitates the degradative activity of the other, and vice versa.

Pfeiffer et al. (2004)'s theory on the evolution of cross-feeding may explain, from an evolutionary viewpoint, why such a syntrophic association became established. The authors proposed the optimization principles that (i) the rate of ATP production is maximized, (ii) the concentration of enzymes of the pathway is minimized, and (iii) the concentration of intermediates of the pathway is minimized. It is un- 
known whether the first principle is valid for our system. The second principle may explain why Sphingomonas sp. TFEE and Burkholderia sp. MN1 are incapable of degrading 3M4N and fenitrothion, respectively. Note that the syntrophic association observed in our system possesses the minimum number of pathways along which fenitrothion is completely degraded (Fig. 2). The cost of degrading 3M4N for Sphingomonas sp. TFEE and that of degrading fenitrothion for Burkholderia sp. MN1 could be too high to generate additional pathways. We observed that high concentrations of 3M4N inhibit the growth of Sphingomonas sp. TFEE in our experiment (data not shown), which highlights the importance of the third optimization principle: minimization of the concentration of intermediates of the pathway. High concentrations of intermediates can be associated with high fitness costs (Dejonghe et al., 2003; Costa et al., 2006). Interestingly, a substantial reduction of 3M4N by Burkholderia sp. MN1 was predicted by our mathematical analysis (Fig. 6D); this is an additional cooperative activity by Burkholderia sp. MN1 within the consortium.

In addition to evolutionary optimization as a reason for the maintenance of syntrophic association, our study revealed the importance of mutualism in the performance of the association and the enhanced ecosystem function arising from the complete degradation of xenobiotics. Cooperation is important for the maintenance of social behavior in microorganisms (Crespi, 2001; Kreft et al., 2005), especially in the formation of a biofilm (Kreft et al., 2004; Hansen et al., 2007). Mutualism plays an important role as a maintenance mechanism of biodiversity and ecosystem functioning, as shown previously in an experiment on the co-dependency of a producer (algae) and decomposer (bacteria) (Naeem et al., 2000). The relationship between biodiversity and the resilience of an ecosystem remains an important topic in ecology (Loreau et al., 2001; Naeem et al., 1997).

The soil environment is exposed to a variety of continuous stresses such as pesticide application. If a particular ecosystem function is conferred with the ability to completely degrade certain organic compounds, the species richness increases when the degradation is mediated by multiple species in syntrophic association rather than a single species. This procedure enhances diversity of species in soil. Syntrophic association becomes increasingly important as a mechanism for the maintenance of a large number of species.

It remains unclear how two bacterial species came to construct syntrophic association whether by loosing a specific gene which is necessary for complete degradation or by getting a gene randomly for unfamiliar pesticide compound in the given environment studied. Horizontal gene transfer (HGT) by mobile genomic elements (MGEs) is known to play a major role in bacterial adaptation to xenobiotic compounds (Top et al., 2003). Plasmids are the most intensively studied catabolic MGEs. The localization of degrading genes on the genomes of Sphingomonas sp. TFEE and Burkholderia sp. MN1 is to be determined to get a clue for understanding these evolutionary consequences. Though we do not refer to the loss and gain of genes, existing Sphingomonas sp. TFEE and Burkholderia sp. MN1 lacked $3 \mathrm{M} 4 \mathrm{~N}$-degrading and fenitrothion-degrading activity, respectively. In conclusion, 
the modeling analyses suggest that Sphingomonas sp. TFEE and Burkholderia sp. MN1 isolated from fenitrothion-treated soil coexist on fenitrothion by syntrophic association, which is characterized by parameters $\beta$ and $\gamma$. Modeling analyses enabled us to reveal the range of parameters of $\beta$ and $\gamma$, which thus explain the growth of Sphingomonas sp. TFEE in experimental soil (Fig. 1), though the population dynamics of the partner Burkholderia sp. MN1 was not chased continuously in the experiment.

\section{A Experimental Methods}

\section{A.1 Soil microcosms}

The soil used for the microcosms was collected from a cornfield in Tochigi prefecture, Japan. The sample was taken from the top $10 \mathrm{~cm}$ of the soil and sieved through a mesh with $2 \mathrm{~mm}$ pore size. The soil was a humic andosol with $\mathrm{pH}$ 7.1, total carbon of $28 \mathrm{mg} \mathrm{kg}^{-1}$ of dry soil, and total nitrogen of $2.3 \mathrm{mg} \mathrm{kg}^{-1}$ of dry soil. Triplicate soil microcosms were prepared by placing moist soil (200 g dry weight) into 500$\mathrm{ml}$ plastic bottles. The microcosms were treated with fenitrothion $\left(50 \mathrm{mg} \mathrm{kg} \mathrm{kg}^{-1}\right.$ of dry soil) at 14-day intervals. Sterile water was added to all samples to adjust the moisture content to $60 \%$ of the maximum water-holding capacity. The soils were then mixed with a spatula, and the microcosms were incubated in the dark at $25^{\circ} \mathrm{C}$.

\section{A.2 Isolation of fenitrothion-degrading and 3M4N-degrading bacteria}

Direct plating, as previously described (Tago et al., 2006), was used for the enumeration and isolation of fenitrothion-degrading bacteria. The agar medium employed for the isolation procedure contained mineral salts, $0.4 \mathrm{~g}$ liter ${ }^{-1}$ yeast extract, and $0.8 \mathrm{~g}$ liter $^{-1}$ fenitrothion emulsion consisting of $50 \%$ fenitrothion (FNT medium) (Hayatsu et al., 2000). Colonies that developed clearing zones on the FNT medium were isolated after the plates had been incubated at $30^{\circ} \mathrm{C}$ for 3 days. The clearing zones are potentially due to fenitrothion transformation. The fenitrothion hydrolase activity was assayed by measuring the formation of 3M4N from fenitrothion as described previously (Tago et al., 2006). 3M4N-degrading bacteria were isolated using direct plating on a Burkholderia-specific PCAT medium (Pallud et al., 2001) containing $0.8 \mathrm{~g} \mathrm{liter}^{-1}$ fenitrothion emulsion. Selected bacteria were streaked onto a nutrient-broth agar medium for purification, and identified on the basis of $16 \mathrm{~S}$ rRNA gene sequences. Crude cell lysates for PCR amplification of the $16 \mathrm{~S}$ rRNA gene sequences were prepared as described previously (Tago et al., 2006). Briefly, the genomic DNA of isolates was extracted by proteinase treatment. The $16 \mathrm{~S}$ rRNA gene sequences for Sphingomonas sp. TFEE and Burkholderia sp. MN1 have been deposited in the DNA Data Bank of Japan (DDBJ) nucleotide sequence database 
under the accession numbers AB381877 and AB381878, respectively.

\section{A.3 Degradation of substrates and bacterial growth}

Cultures of Sphingomonas sp. TFEE and Burkholderia sp. MN1 grown in a mineral salts medium containing $0.5 \mathrm{~g} \mathrm{liter}^{-1}$ glucose, $1.0 \mathrm{~g}$ liter $^{-1}$ tryptone, and $0.5 \mathrm{~g} \mathrm{liter}^{-1}$ yeast extract (1/2 GPY medium) were pelleted, washed, and suspended in mineral salts medium. The bacterium were cultured in mineral salts medium supplemented with 0 to $1.0 \mathrm{mM}$ of fenitrothion, 3M4N, or MHQ as a sole carbon source. The initial inoculum density was approximately $5 \times 10^{7}$ cells $\mathrm{ml}^{-1}$ culture. The cultures were shaken at $150 \mathrm{rpm}$ for 24 to $48 \mathrm{~h}$ at $30^{\circ} \mathrm{C}$. Growth was determined by measuring the optical density at $600 \mathrm{~nm}\left(\mathrm{OD}_{600}\right)$ or enumerating $\mathrm{CFU} \mathrm{m}{ }^{-1}$. Metabolites of substrates were identified by reverse-phase high-performance liquid chromatography (HPLC) analysis with a diode array detector using a $\mathrm{C}_{18}$ column (Hayatsu et al., 2000).

\section{A.4 Co-culture of the two bacteria}

For co-culture with MHQ, the two bacteria were cultured in mineral salts medium supplemented with $0.05 \mathrm{~g} \mathrm{liter}^{-1}$ yeast extract and $0.1 \mathrm{mM}$ of MHQ. The initial inoculum density was approximately $1 \times 10^{7}$ cells $\mathrm{ml}^{-1}$ culture. The cultures were shaken at $150 \mathrm{rpm}$ for $28 \mathrm{~h}$ at $30^{\circ} \mathrm{C}$. As soon as MHQ was consumed, a $0.1 \mathrm{mM}$ of MHQ was added to the medium, making a total of $1.0 \mathrm{mM}$ of added MHQ. The MHQ concentration in the medium was measured by HPLC. For each sample, $\mathrm{OD}_{600}$ was measured over time, and the number of each bacteria was enumerated (in CFU $\mathrm{ml}^{-1}$ ) after $25 \mathrm{~h}$ of incubation.

For co-culture with fenitrothion, the two bacteria were cultured in mineral salts medium supplemented with $0.5 \mathrm{mM}$ of fenitrothion. The initial inoculum density ranged from $1 \times 10^{4}$ cells ml ${ }^{-1}$ culture to $1 \times 10^{8}$ cells ml $^{-1}$ culture. The cultures were shaken at $150 \mathrm{rpm}$ for $48 \mathrm{~h}$ at $30^{\circ} \mathrm{C}$. The number of each bacteria was enumerated (in $\mathrm{CFU} \mathrm{ml} \mathrm{m}^{-1}$ ) after $25 \mathrm{~h}$ of incubation.

\section{B Analysis of Experiments}

\section{B.1 The existence conditions for coexistence equilibria}

Components of the syntrophic consortium equilibrium are obtained as a positive constant solution of $(\mathrm{Eq})$. It follows from the fifth equation of $(\mathrm{Eq})$ that the concentration of MHQ is given by $S_{2}^{*}=\frac{a_{1} \mu_{1}}{m_{1}-\mu_{1}}$ if $m_{1}>\mu_{1}$. It follows from the fourth equation of $(\mathrm{Eq})$ that the concentration of $3 \mathrm{M} 4 \mathrm{~N}$ is given by $S_{1}^{*}=\frac{a_{2} \mu_{2}}{m_{2}(1-\gamma)-\mu_{2}}$ if $\gamma<\gamma_{\max }$, where $\gamma_{\max }=1-\frac{\mu_{2}}{m_{2}}$. The concentration of Sphingomonas sp. TFEE at the syntrophic consortium equilibrium is given by a positive root of the following 
quadratic equation

$$
c_{0} x_{1}^{2}+c_{1} x_{1}+c_{2}=0
$$

where

$$
c_{0}=\frac{\beta \mu_{1}}{\eta_{1}}, \quad c_{1}=\frac{d_{0} \mu_{1}}{\eta_{1}}+\beta\left(\gamma d_{1} S_{1}^{*}+d_{2} S_{2}^{*}\right)-\lambda \beta \gamma \text { and } c_{2}=d_{0}\left(\gamma d_{1} S_{1}^{*}+d_{2} S_{2}^{*}\right) .
$$

If $c_{1}<0$ and $c_{1}^{2}-4 c_{0} c_{2}>0$, there exists a positive constant $x_{1}^{+}$such that $x_{1}^{+}$corresponds to the density of Sphingomonas sp. TFEE. Accordingly, the concentration of fenitrothion and the density of Burkholderia sp. MN1 at the syntrophic consortium equilibrium are given by $S_{0}^{+}=\frac{\lambda}{d_{0}+\beta x_{1}^{+}}$and $x_{2}^{+}=\frac{1-\gamma}{\gamma} \frac{\eta_{2}}{\mu_{2}}\left(d_{2} S_{2}^{*}+\frac{\mu_{1}}{\eta_{1}} x_{1}^{+}\right)$, respectively. Note that the syntrophic consortium equilibrium is likely to exist for sufficiently large $\beta$ when natural loss rate of two bacteria $\mu_{j}(j=1,2)$ and the natural decomposition rate of substrates $d_{i}(i=0,1,2)$ are very small compared with the other parameters. The default parameters satisfy this situation.

\section{B.2 Local stability of equilibria}

Local stability conditions for null degradation equilibrium $E_{N}$ and syntrophic consortium equilibrium $E_{S}$ are investigated. The Jacobi matrix associated with the linearized equations of $(\mathrm{Eq})$ defined at $E_{N}$ is given by

$$
\mathbf{J}_{\mathbf{N}}:=\left(\begin{array}{ccccc}
-d_{0} & 0 & 0 & -\beta \frac{\lambda}{d_{0}} & 0 \\
0 & -d_{1} & 0 & \beta \frac{\lambda}{d_{0}} & 0 \\
0 & 0 & -d_{2} & 0 & 0 \\
0 & 0 & 0 & -\mu_{1} & 0 \\
0 & 0 & 0 & 0 & -\mu_{2}
\end{array}\right)
$$

The corresponding characteristic equation $p_{N}(r):=\operatorname{det}\left(r I-J_{N}\right)=0$ is

$$
p_{N}(r)=\left(r+d_{0}\right)\left(r+d_{1}\right)\left(r+d_{2}\right)\left(r+\mu_{1}\right)\left(r+\mu_{2}\right)=0 .
$$

Hence $E_{N}$ is always locally stable. Hereafter we assume that the conditions for the existence of $E_{S}$ derived in Appendix B.1 are satisfied. The Jacobi matrix associated with the linearized equatons of (Eq) defined at syntrophic consortium equilibrium $E_{S}$ is given by

$$
\mathbf{J}_{\mathbf{S}}:=\left(\begin{array}{ccccc}
-d_{0}-\beta x_{1}^{*} & 0 & 0 & -\beta S_{0}^{*} & 0 \\
\beta x_{1}^{*} & -d_{1}-f_{2}^{\prime}\left(S_{1}^{*}\right) \frac{x_{2}^{*}}{\eta_{2}} & 0 & \beta S_{0}^{*} & -\frac{\mu_{2}}{(1-\gamma) \eta_{2}} \\
0 & \gamma f_{2}^{\prime}\left(S_{1}^{*}\right) \frac{x_{2}^{*}}{\eta_{2}} & -d_{2}-f_{1}^{\prime}\left(S_{2}^{*}\right) \frac{x_{1}^{*}}{\eta_{1}} & -\frac{\mu_{1}}{\eta_{1}} & \frac{\gamma \mu_{2}}{(1-\gamma) \eta_{2}} \\
0 & 0 & f_{1}^{\prime}\left(S_{2}^{*}\right) x_{1}^{*} & 0 & 0 \\
0 & (1-\gamma) f_{2}^{\prime}\left(S_{1}^{*}\right) x_{2}^{*} & 0 & 0 & 0
\end{array}\right),
$$


where $f_{i}^{\prime}\left(S_{j}\right)=\frac{m_{i} a_{i}}{\left(a_{i}+S_{j}\right)^{2}}(i=1,2, j=0,1,2)$. The characteristic equation $p_{S}(r):=$ $\operatorname{det}\left(r I-J_{S}\right)=0$ can determine the local stability of $E_{S}$. Note that $p_{S}(r)$ is a fifth-order polynomial of the form

$$
p_{S}(r)=r^{5}+a_{1} r^{4}+a_{2} r^{3}+a_{3} r^{2}+a_{4} r+a_{5} .
$$

Each coefficient $a_{i}(i=1,2, \cdots, 5)$ can be explicitly obtained by expanding $\operatorname{det}(r I-$ $\left.J_{S}\right)$. Although the Routh-Hurwitz criterion provides a versatile way to determine the local stability of $E_{S}$, algebraic relations among $a_{i}$ s are generally complicated. It is one of the possible choices to confirm the Routh-Hurwitz criterion by implementing symbolic calculations for $\operatorname{det}\left(r I-J_{S}\right)$ to obtain the explicit expression of $a_{i}$, following which the Routh array is numerically calculated. However, this method is not useful if the problem in consideration is to solve a high-order polynomial equation. Another useful way is to obtain numerical approximate solutions of $p_{S}(r)=0$ directly. One of the versatile free software, GNU Octave, can provide a numerical environment to implement an algorithm for solving (2) directly. The predefined function "eig" in Octave returns numerical approximate solution of the characteristic equation $p_{S}(r)=0$. The underlying algorithm to obtain numerical approximation in Octave is based on Schur decomposition of a matrix (see a standard textbook of matrix computation). In summary, using Octave enables us to confirm the local stability of $E_{S}$ easily.

For example, the default parameter with $\beta=5$ and $\gamma=0.5$ is adopted to confirm whether $E_{S}$ is locally stable. The eigenvalues of $p_{S}(r)=0$ are numerically given by $r_{1}=-12.19942<0, r_{2}=-6.59079<0, r_{3}=-2.98849<0, r_{4}=-0.01980<0$ and $r_{5}=-0.00991<0$, implying that $E_{S}$ is locally stable. We constructed a program which enables us to check the stability of $E_{S}$ by varying one of parameters. We confirmed that $E_{S}$ is locally stable whenever it exists, as long as we performed numerical computations.

\section{B.3 Mathematical model for MHQ-supplemented culture}

Note that the population growth of Sphingomonas sp. TFEE in co-culture is less than that of in mono-culture. This suggests that exploitative competition for MHQ as a limiting energy source between two bacteria may occur. We constructed a mathematical model that describes the growth of two bacteria in an MHQ-treated medium. We assume that MHQ is continuously supplied to the culture at a constant rate, denoted by $\lambda_{\mathrm{MHQ}}$. The model is given by

$$
\begin{aligned}
& \frac{d S_{2}}{d t}=\lambda_{\mathrm{MHQ}}-d_{2} S_{2}-f_{1}\left(S_{2}\right) \frac{x_{1}}{\eta_{1}}-f_{2}\left(S_{2}\right) \frac{x_{2}}{\eta_{2}}, \\
& \frac{d x_{1}}{d t}=x_{1}\left(f_{1}\left(S_{2}\right)-\mu_{1}\right), \\
& \frac{d x_{2}}{d t}=x_{2}\left(f_{2}\left(S_{2}\right)-\mu_{2}\right) . \\
& 16
\end{aligned}
$$


MHQ was treated totally ten times during 25 hours. $0.1 \mathrm{mM}$ of MHQ is supplied in each treatment. Thus we set $\lambda_{\mathrm{MHQ}}=0.04 \mathrm{mM}$ per hour. Since the experiment was performed during 25 hours which is very short compared with the experiment in soil microcosm, we shall use "hour" as the unit of time. Then the values of the default parameters are simultaneously translated into the time scale of the MHQ experiment. The growth kinetics of two bacteria may differ between soil microcosm and MHQ-treated medium. We therefore choose the value of $m_{j}$ and $a_{j}(j=1,2)$ independent of the default parameters to obtain the correspondence with the experimental data in mono- and co-culture treated with MHQ at 25 hours (Table 1 and Fig. 3).

A mathematical model for each species in mono-culture is obtained by setting $x_{1}(0)=0$ or $x_{2}(0)=0$. Numerical calculations were implemented using the initial conditions $S_{2}(0)=0$ and $x_{1}(0)=x_{2}(0)=10^{7}$ cell $\mathrm{ml}^{-1}$. Although another choice of parameters can realize the same situation observed in the MHQ experiment, the numerical calculation result shows a good correspondence to the MHQ experiment (see Fig. 3 and Fig. 8). We can predict the long time dynamics of two bacterial populations in the MHQ-treated media. According to the theory of the chemostat, the only species with the lowest break-even concentration can survive (Smith et al., 1994). On model (3), the break-even concentrations for Sphingomonas sp. TFEE and Burkholderia sp. MN1 are given by $\Lambda_{\mathrm{TFEE}}=1.67 \times 10^{-4}$ and $\Lambda_{\mathrm{MN} 1}=4.87 \times 10^{-4}$, respectively. Hence only Sphingomonas sp. TFEE may eventually survive if MHQ is continuously supplied to the media for more than 25 hours. In other words, long-term coexistence of two bacteria in equilibrium is never expected as long as the energy source for two bacteria is limited to MHQ alone. Hence the manner of resource utilization by forming the syntrophic association is essential for the longterm coexistence of two bacteria.

\section{B.4 A variant of model (Eq)}

We shall investigate the dynamics exhibited by a variant of (Eq) in which Burkholderia sp. MN1 is allowed to utilize MHQ which has been distributed in soil by itself. The variant model is given by

$$
\begin{aligned}
\frac{d S_{0}}{d t} & =\lambda-d_{0} S_{0}-\beta S_{0} x_{1} \\
\frac{d S_{1}}{d t} & =-d_{1} S_{1}+\beta S_{0} x_{1}-f_{2}\left(S_{1}\right) \frac{x_{2}}{\eta_{2}} \\
\frac{d S_{2}}{d t} & =-d_{2} S_{2}+\gamma f_{2}\left(S_{1}\right) \frac{x_{2}}{\eta_{2}}-f_{1}\left(S_{2}\right) \frac{x_{1}}{\eta_{1}}-f_{2}\left(S_{2}\right) \frac{x_{2}}{\eta_{2}} \\
\frac{d x_{1}}{d t} & =x_{1}\left(f_{1}\left(S_{2}\right)-\mu_{1}\right), \\
\frac{d x_{2}}{d t} & =x_{2}\left((1-\gamma) f_{2}\left(S_{1}\right)+f_{2}\left(S_{2}\right)-\mu_{2}\right) .
\end{aligned}
$$


Since the exploitative competition for MHQ between two bacteria in a soil microcosm is present, it is necessary to impose additional conditions to ensure the successful establishment of the syntrophic association. We infer that Sphingomonas sp. TFEE is superior to Burkholderia sp. MN1 in the exploitative competition for MHQ. As introduced in Appendix B.3, the break-even concentrations play an important role. The condition $\Lambda_{\mathrm{TFEE}}<\Lambda_{\mathrm{MN} 1}$ is additionally required for the existence of syntrophic consortium equilibrium $E_{S}$. Fig. 9 depicts the same diagram to Fig. 5 which convinces that model (Eq') exhibits qualitatively the same outcome to model (Eq). The syntrophic association can be robustly established even though there exists an exploitative competition between two bacteria on the common limiting resource MHQ.

\section{Acknowledgments}

This work was partly supported by the Global Environment Research Fund of Ministry of the Environment, Japan. SN was supported by (i) the Sasakawa Scientific Research Grant from The Japan Science Society and (ii) Research Fellowships of the Japan Society for the Promotion of Science for Young Scientists. We thank two anonymous reviewers for their useful comments.

\section{References}

Alonso, J.L., Sabater, C., Ibañez, M.J., Amoros, I., Botella, M.S., Carrasco, J., 1997. Fenitrothion and 3-methyl-4-nitrophenol degradation by two bacteria in natural waters under laboratory conditions. J. Environ. Sci. Heal. A32, 799-812.

Armstrong, R.A., McGehee, R., 1980. Competitive exclusion. Am. Nat. 115, 151170.

Bratbak G., Thisstad T.F., 1985. Phytoplankton-bacteria interactions: an apparent paradox? Analysis of a model system with both competition and commensalism. Mar. Ecol. Prog. Ser. 25, 23-30.

Costa, E., Pérez, J., Kreft, J-U., 2006. Why is metabolic labour divided in nitrification? Trends Microbiol. 14, 213-219., doi:10.1016/j.tim.2006.03.006.

Crespi, B.J., 2001. The evolution of social behavior in microorganisms. Trends Ecol. Evol. 16, 178-183., doi:10.1016/S0169-5347(01)02115-2.

Dejonghe, W., Berteloot, E., Goris, J., Boon, N., Crul, K., Maertens, S., Hofte, M., Vos, P.D., Verstraete, W., Top, E.M., 2003. Synergistic degradation of linuron by a bacterial consortium and isolation of a single linuron-degrading Variovorax strain. Appl. Environ. Microbiol. 69, 1532-1541. 
Doebeli, M., 2002. A model for the evolutionary dynamics of cross-feeding polymorphisms in microorganisms. Popul. Ecol. 44, 59-70.

Friesen, M.L., Saxer, G., Travisano, M., Doebeli, M., 2004. Experimental evidence for sympatric ecological diversification due to frequency-dependent competition in Escherichia coli. Evolution 58, 245-260.

Gans, J., Wolinsky, M., Dunbar, J., 2005. Computational improvements reveal great bacterial diversity and high metal toxicity in soil. Science 309, 1387-1390.

Grover J.P., 1997. Resource competition. Springer, New York.

Hansen, S.K., Rainey, P.B., Haagensen, J.A.J., Molin, S., 2007. Evolution of species interactions in a biofilm community. Nature 445, 533-536.

Hayatsu, M., Hirano, M., Tokuda, S., 2000. Involvement of two plasmids in fenitrothion degradation by Burkholderia sp. NF100. Appl. Environ. Microbiol. 66, $1737-1740$.

Keener, J.P., Sneyd, J., 1998. Mathematical Physiology. Springer, New York.

Keller, L., Surette, M.G., 2006. Communication in bacteria: an ecological and evolutionary perspective. Nat. Rev. Microbiol. 4, 249-258.

Kreft, J-U., 2004. Biofilms promote altruism. Microbiology 150, 2751-2700.

Kreft, J-U., Bonhoeffer, S., 2005. The evolution of groups of cooperating bacteria and the growth rate versus yield trade-off. Microbiology 151, 637-641.

Loreau, M., Naeem, S., Inchausti, P., Bengtsson, J., Grime, J.P., Hector, A., Hooper, D.U., Huston, M.A., Raffaelli, D., Schmid, B., Tilman, D., Wardle, D.A., 2001. Biodiversity and ecosystem functioning: current knowledge and future challenges. Science 294, 804-808.

Madigan, M.T., Martinko, J.M., Parker, J., 2000. Biology of Microorganisms, Ninth edition. Prentice Hall, Inc., New Jersey, p. 574, 624-626, 647.

Miki, T., Yamamura, N., 2005. Theoretical model of interactions between particleassociated and free-living bacteria to predict functional composition and succession in bacterial communities. Aquat. Microb. Ecol. 39, 35-46.

Naeem, S., Li, S., 1997. Biodiversity enhances ecosystem reliability. Nature 390, $507-509$.

Naeem, S., Hahn, D.R., Schuurman, G., 2000 Producer-decomposer co-dependency influences biodiversity effects. Nature 403, 762-754. 
Overmann J., Schubert, K., 2002. Phototrophic consortia: model systems for symbiotic interrelations between prokaryotes. Arch. Microbiol. 177, 201-208.

Pallud, C., Viallard, V., Balandreau, J., Normand, P., Grundmann, G., 2001. Combined use of a specific probe and PCAT medium to study Burkholderia in soil. J. Microbiol. Methods 47, 25-34., doi:10.1016/S0167-7012(01)00287-1.

Pfeiffer, T., Bonhoeffer, S., 2004. Evolution of cross-feeding in microbial populations. Am. Nat. 163, E126-E135.

Raghoebarsing, A.A., Pol, A., Pas-Schoonen, K.T., Smolders, A.J.P., Ettwig, K.F., Rijpstra, W.I.C., Schouten, S., Damsté, J.S.S., Camp, H.J.M.O., Jetten, M.S.M., Strous, M., 2006. A microbial consortium couples anaerobic methane oxidation to denitrification. Nature 440, 918-921.

Rainey, P.B., Rainey, K., 2003. Evolution of cooperation and conflict in experimental bacterial populations. Nature 425, 72-74.

Rosenzweig, R.F., Sharp, R.R., Treves, D.S., Adams, J., 1994. Microbial evolution in a simple unstructured environment: genetic differentiation in Escherichia coli. Genetics 137, 903-917.

Schink, B., 1997. Energetics of syntrophic cooperation in methanogenic degradation. Microbiol. Mol. Biol. Rev. 61, 262-280.

Smith, H.L., Waltman, P., 1994. The theory of the chemostat: dynamics of microbial competition. Cambridge University Press, New York.

Smith, D., Alvey, S., Crowley, D.E., 2005. Cooperative catabolic pathways within an atrazine-degrading enrichment culture isolated from soil. FEMS Microbiol. Ecol. 53, 265-273., doi:10.1016/j.femsec.2004.12.011.

Tago, K., Sekiya, E., Kiho, A., Katsuyama, C., Hoshito, Y., Yamada, N., Hirano, K., Sawada, H., Hayatsu, M., 2006. Diversity of fenitrothion-degrading bacteria in soils from distant geographical areas. Microbes Environ. 21, 58-64.

Top, E.M., Springael, D., 2003. The role of mobile genetic elements in bacterial adaptation to xenobiotic organic compounds. Curr. Opin. Biotechnol. 14, 262269.

Torsvik, V., Øvreås, L., Thingstad, T. F., 2002. Prokaryotic diversity-Magnitude, dynamics, and controlling factors. Science 296, 1064-1066.

Torsvik, V., Øvreås, L., 2002. Microbial diversity and function in soil: from genes to ecosystems. Curr. Opin. Microbiol. 5, 240-245.

Valentine D.L., 2002. Biogeochemistry and microbial ecology of methane oxidation in anoxic environments: a review. Antonie Van Leeuwenhoek 81, 271-282. 
Whitman, W.B., Coleman, D.C., Wiebe, W.J., 1998. Prokaryotes: The unseen majority. Proc. Natl. Acad. Sci. USA. 95, 6578-6583.

Zissi, U., Lyberatos, G., 2001. Partial degradation of $p$-Aminoazobenzene by a defined mixed culture of Bacillus subtilis and Stenotrophomonas maltophilia. Biotechnol. Bioeng. 72, 49-54. 
Table 1: Growth of Sphingomonas sp. TFEE and Burkholderia sp. MN1 in MHQsupplemented culture experiments

\begin{tabular}{cccc}
\hline Mono-culture & \multicolumn{2}{c}{ Bacterial numbers $\left(10^{8} \mathrm{cfu} \mathrm{ml}^{-1}\right)$} \\
& Time & TFEE & MN1 \\
\hline without MHQ & $0 \mathrm{~h}$ & $0.146 \pm 0.007$ & $0.133 \pm 0.008$ \\
& $25 \mathrm{~h}$ & $2.45 \pm 0.14$ & $1.53 \pm 0.05$ \\
with MHQ & $0 \mathrm{~h}$ & $0.126 \pm 0.013$ & $0.129 \pm 0.005$ \\
& $25 \mathrm{~h}$ & $20.6 \pm 2.1$ & $7.5 \pm 1.1$ \\
\hline Co-culture & \multicolumn{3}{c}{ Bacterial numbers $\left(10^{8} \mathrm{cfu} \mathrm{ml}^{-1}\right)$} \\
& Time & TFEE & $\mathrm{MN} 1$ \\
\hline without MHQ & $0 \mathrm{~h}$ & $0.136 \pm 0.016$ & $0.139 \pm 0.008$ \\
& $25 \mathrm{~h}$ & $1.13 \pm 0.11$ & $1.09 \pm 0.16$ \\
with MHQ & $0 \mathrm{~h}$ & $0.128 \pm 0.013$ & $0.125 \pm 0.013$ \\
& $25 \mathrm{~h}$ & $5.9 \pm 0.6$ & $4.8 \pm 0.2$ \\
\hline
\end{tabular}

Table 2: Notation summary

\begin{tabular}{ccc}
\hline Symbol & Definition & Unit \\
\hline$S_{0}, S_{1}, S_{2}$ & concentrations of substrates & $\mathrm{mM}$ \\
$x_{1}, x_{2}$ & densities of bacteria & $10^{7} \mathrm{cells} \mathrm{g}^{-1}$ \\
$f_{j}\left(S_{i}\right)$ & substrate-specific uptake & - \\
$m_{1}, m_{2}$ & maximum uptake rates & day $^{-1}$ \\
$a_{1}, a_{2}$ & half-saturation constants & $\mathrm{mM}$ \\
$\eta_{1}, \eta_{2}$ & substrate yields of bacteria & - \\
$\mu_{1}, \mu_{2}$ & bacteria mortalities & day $^{-1}$ \\
$\lambda$ & supply rate of fenitrothion & $\mathrm{mM} \mathrm{day}^{-1}$ \\
$d_{0}, d_{1}, d_{2}$ & substrate decomposition rates & day $^{-1}$ \\
$\beta$ & fenitrothion-degradation rate & {$\left[10^{7}\right.$ cells $^{-1}$ day $^{-1}$} \\
$\gamma$ & fraction of MHQ distribution & - \\
\hline
\end{tabular}




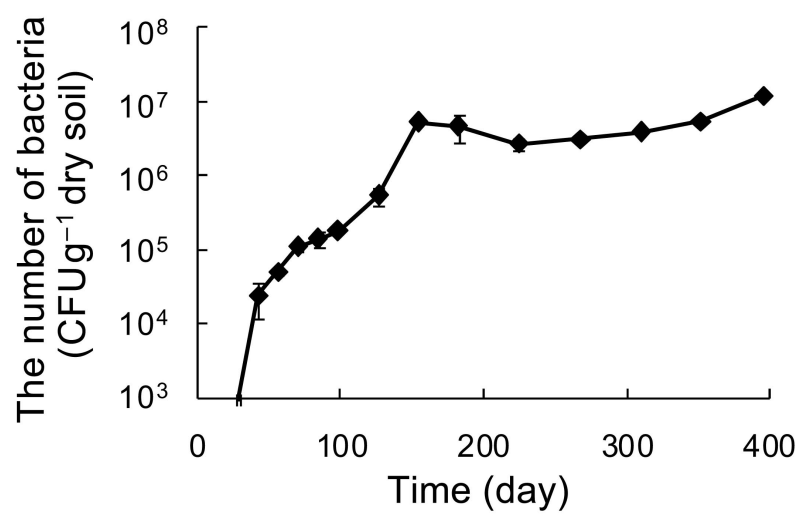

Figure 1: The number of fenitrothion-degrading bacteria (i.e., Sphingomonas sp. TFEE) in the soil microcosm. The data points show the means based on data from triplicate measurements. The initial number of Sphingomonas sp. TFEE was below the detection limit $\left(10^{3}\right.$ cells $\left.\mathrm{g}^{-1}\right)$.

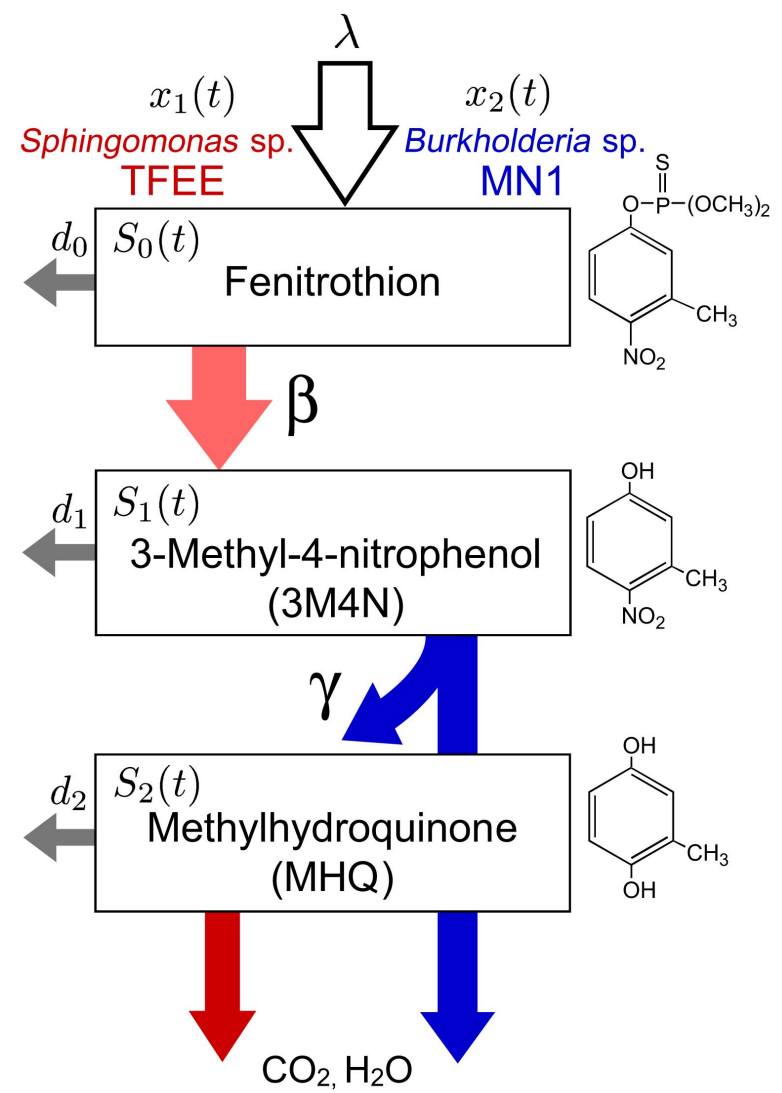

Figure 2: Schematic representation of fenitrothion degradation by Sphingomonas sp. TFEE and Burkholderia sp. MN1. Definition of parameters is given in Table 2. 


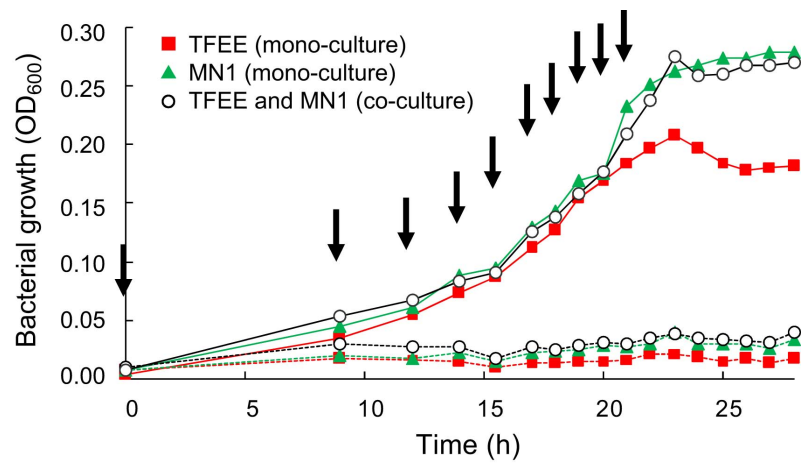

Figure 3: Growth of Sphingomonas sp. TFEE and Burkholderia sp. MN1 in monoand co-culture, as measured by $\mathrm{OD}_{600}$ in cultures fed with MHQ (solid lines) or without MHQ (dashed lines). An additional $0.1 \mathrm{mM}$ of MHQ was fed at the points indicated by black arrows. 

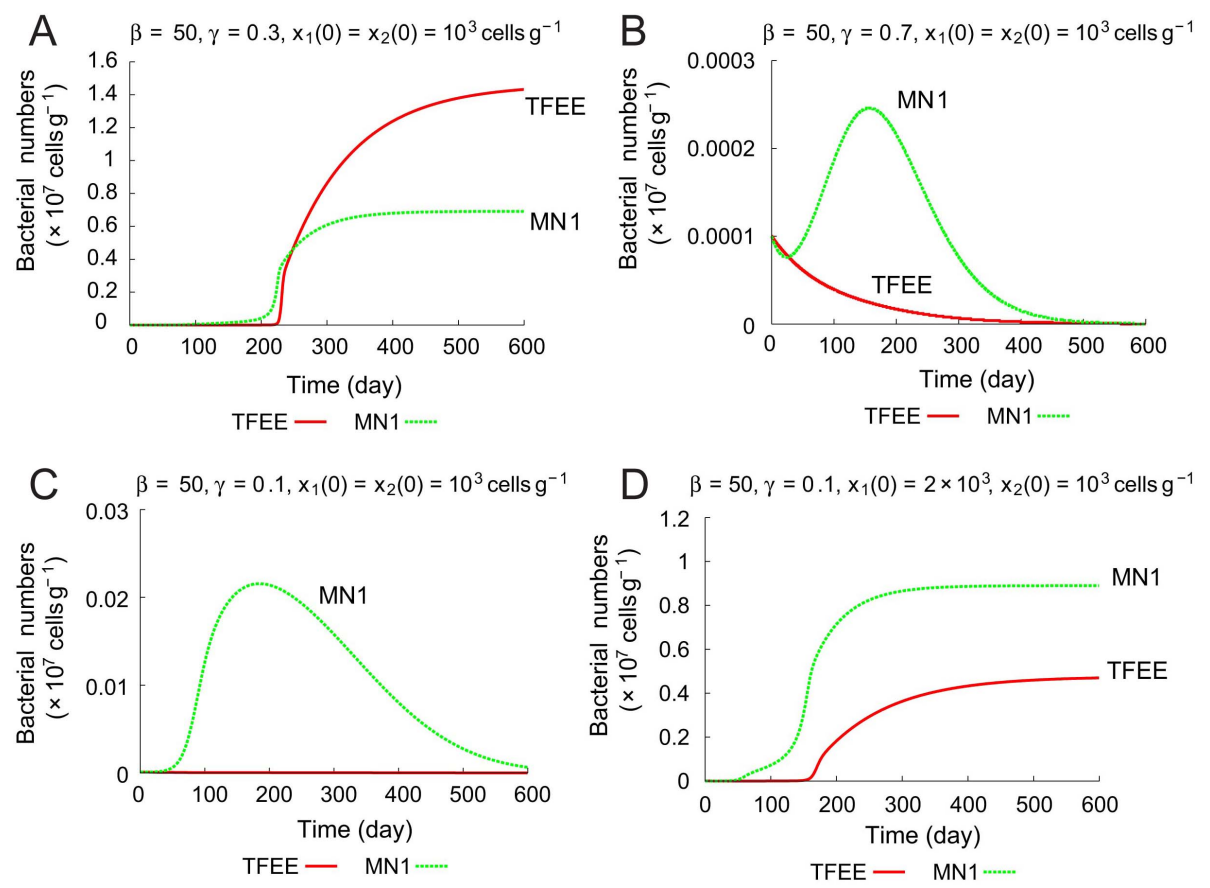

Figure 4: Numerical calculation results for (A) $\beta=50$ and $\gamma=0.3$, (B) $\beta=50$ and $\gamma=0.7$, (C) $\beta=50$ and $\gamma=0.1$ with the default initial population size for two species, and (D) $\beta=50$ and $\gamma=0.1$ with the twice elevated initial population size of Sphingomonas sp. TFEE; $x_{1}(0)=2 \times 10^{3}$ cells $\mathrm{g}^{-1}$. Solid-red lines: Sphingomonas sp. TFEE. Dashed-green lines: Burkholderia sp. MN1. (A) Two bacteria can grow substantially by forming syntrophic association. (B) Both of two bacteria do not exhibit substantial growth (less than $3 \times 10^{3}$ cells g $^{-1}$ ) and fall into under the detectable level. (C) Only Burkholderia sp. MN1 exhibits significant but temporal growth. This result indicates that Burkholderia sp. MN1 will eventually decline under the detectable level due to the breakdown of the syntrophic association with Sphingomonas sp. TFEE. (D) Both two bacteria exhibit significant growth, suggesting that the augmentation of Sphingomonas sp. TFEE may restore the syntrophic association. 


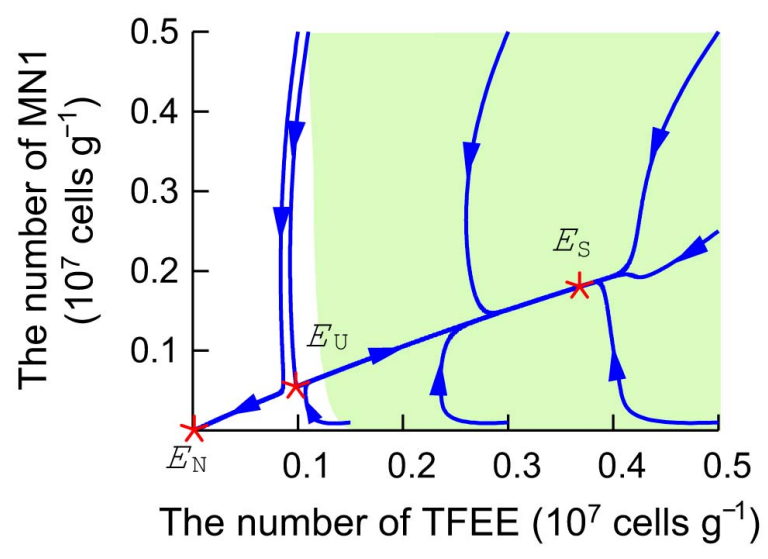

Figure 5: Eight representative trajectories (blue curves) starting at different initial population size. $\beta=0.05$ and $\gamma=0.3$. Seven trajectories on the associative region (green) converge to syntrophic consortium equilibrium $E_{S}$, representing successful degradation of fenitrothion. One trajectory on the dissociative region (white) converges to degradation free equilibrium $E_{N}$, representing the fail of degradation. Three $*$-marks indicate the location of equilibria.
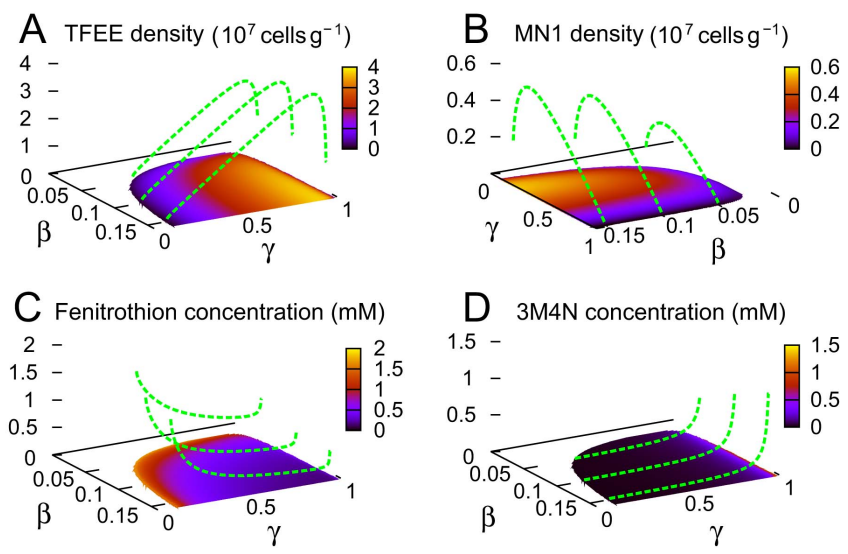

Figure 6: Equilibrium densities/concentrations of (A) Sphingomonas sp. TFEE, (B) Burkholderia sp. MN1, (C) fenitrothion, and (D) 3M4N in the successful degradation of fenitrothion. In each panel, equilibrium density/concentration is mapped onto the $\beta \gamma$-plane with three representative equilibrium curves (dashed-green lines). In each panel, the maximum density/concentration attained at the top of the dashed-green lines corresponds to the brightest region in contour plot. 

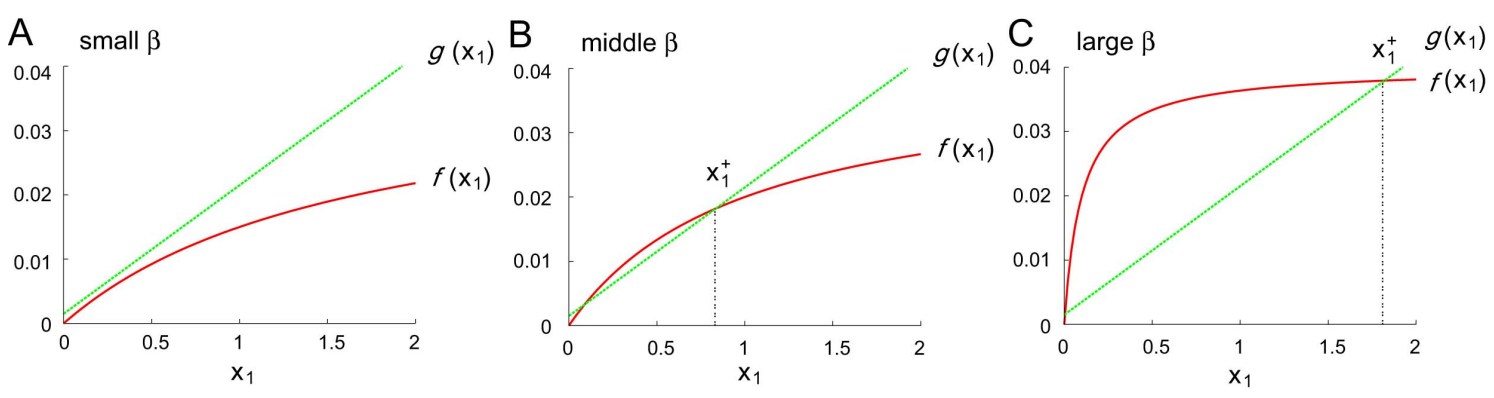

Figure 7: Two positive equilibria are derived from the intersection points of $f\left(x_{1}\right)=$ $\frac{\lambda \gamma \beta x_{1}}{d_{0}+\beta x_{1}}$ and $g\left(x_{1}\right)=\frac{\mu_{1}}{\eta_{1}} x_{1}+\gamma d_{1} S_{1}^{*}+d_{2} S_{2}^{*}$. The intersection points do not exist for (A) small $\beta$, while they exist for (B) middle $\beta$ and (C) large $\beta$. Large $\beta$ facilitates the existence of positive equilibria; $\beta$ is essential to the establishment of syntrophic association.

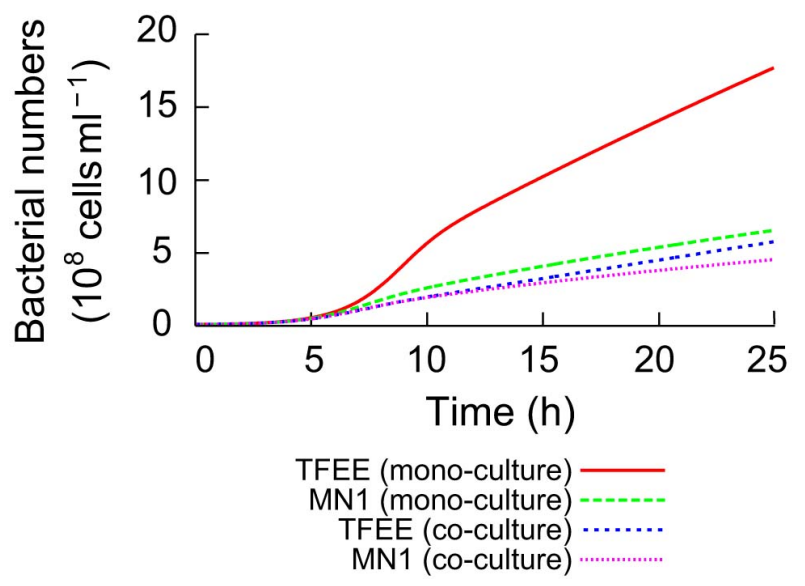

Figure 8: Mathematical representation of the mono- and co-culture of two bacteria in MHQ-supplemented culture, showing the growth of Sphingomonas sp. TFEE in mono-culture (solid red line), Burkholderia sp. MN1 in mono-culture (dashed green line), Sphingomonas sp. TFEE in co-culture (dotted blue line) and Burkholderia sp. MN1 in co-culture (small dotted pink line). The values of parameters are $\lambda_{\mathrm{MHQ}}=0.04, d_{2}=0.00208 m_{1}=0.4, a_{1}=0.16, \mu_{1}=0.0004166, \eta_{1}=0.5$, $m_{2}=0.6, a_{2}=0.35, \mu_{2}=0.000833, \eta_{2}=0.2$. 


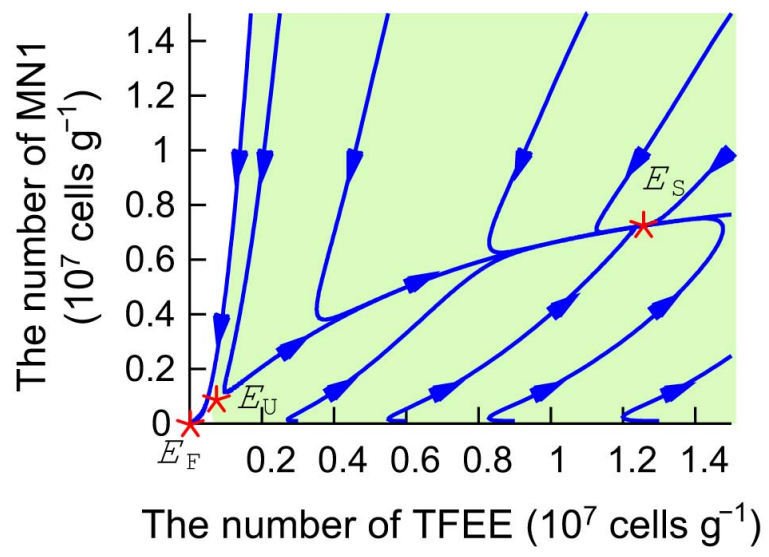

Figure 9: The same diagram to Figure 5 which is obtained by performing numerical calculations to a variant model (Eq'). Ten representative trajectories (blue curves) starting at different initial points. $\beta=0.1$ and $\gamma=0.6$. 